\title{
SCALING LAWS FOR DROPLETS SPREADING UNDER CONTACT-LINE FRICTION*
}

\author{
MARIA CHIRICOTTO ${ }^{\dagger}$ AND LORENZO GIACOMELLI ${ }^{\ddagger}$
}

\begin{abstract}
This manuscript is concerned with the spreading of a liquid droplet on a plane solid surface. The focus is on effective conditions which relate the speed of the contact line (where liquid, solid, and vapor meet) to the microscopic contact angle. One such condition has been recently proposed by Weiqing Ren and Weinan E [Phys. Fluids 19, 022101, 2007]: it includes into the model the effect of frictional forces at the contact line, which arise from unbalanced components of the Young's stress. In lubrication approximation, the spreading of thin droplets may be modeled by a class of free boundary problems for fourth order nonlinear degenerate parabolic equations. For speed-dependent contact angle conditions of rather general form, a matched asymptotic study of these problems is worked out, relating the macroscopic contact angle to the speed of the contact line. For the specific model of Ren and E, ODE arguments are then applied to infer the intermediate scaling laws and their timescales of validity: in complete wetting, they depend crucially on the relative strength of surface friction (at the liquid-solid interface) versus contact-line friction; in partial wetting, they also depend on the magnitude of the static contact-angle.
\end{abstract}

Key words. Moving contact line, droplets, thin film equations, fourth order degenerate parabolic equations, free boundary problems, lubrication theory, matched asymptotic expansions, intermediate scaling laws.

AMS subject classifications. 35Bxx, 35K30, 35K65, 76A20, 76D08.

\section{Introduction and results}

1.1. The model. Understanding the dynamics of wetting phenomena of droplets on solid substrates is still an ongoing challenge. The difficulty comes from the classical theory of fluids. Indeed, in the Navier-Stokes equations, the constant viscosity coupled with a no-slip boundary condition at the liquid-solid interface results in a nonphysical singularity at moving contact lines, i.e. an infinite rate of energy dissipation $[19,10]$. Many models have been proposed in order to remove this singularity (see e.g. $[9,21,5])$. All of them introduce at least one "microscopic" lengthscale in the problem. The most common approach is to introduce effective slip conditions at the liquid-solid interface; the simplest slippage model, the so-called Navier slip, reads as

$$
U=\mu B U_{\zeta} \quad \text { at the liquid-solid interface, } \zeta=0 .
$$

Here we adopt a two-dimensional framework, $(\xi, \zeta) \in \mathbb{R} \times \mathbb{R}_{+}$with the solid substrate at $\zeta=0, U$ denotes the horizontal component of the velocity field within the liquid phase, $\mu$ denotes the liquid's viscosity and $\mu B \geq 0$ is the so-called slip length. The ratio $1 / B$ is to be understood as a friction coefficient between the liquid and the solid.

Away from the contact line where the liquid, the solid and the surrounding vapor meet, slippage models for single-phase flows have survived an extensive crosscheck by MD simulations (see e.g. [27] and the discussion in [22, 23]). However, recent

*Received: December 15, 2011; accepted (in revised form): June 7, 2012. Communicated by Weinan E.

${ }^{\dagger}$ Dipartimento di Scienze di Base e Applicate per l'Ingegneria, Sapienza Università di Roma, Italy (maria.chiricotto@sbai.uniroma1.it).

${ }_{\ddagger}^{\ddagger}$ Dipartimento di Scienze di Base e Applicate per l'Ingegneria, Sapienza Università di Roma, Italy (lorenzo.giacomelli@sbai.uniroma1.it). 
investigations by Qian, Wang, and Sheng [22] and by Ren and E [23] have confirmed that, near the contact line region, slippage models such as (1.1) cease to provide a valid description of the dynamics; there, the main driving force which is responsible for the slip is the unbalanced Young's stress. Of particular interest in this note is the contribution by Ren and E [23] and by Ren, Hu, and E [25] (see also [24]). There, by a combination of molecular dynamics and continuum thermodynamics, an effective continuum model is derived, in which the unbalanced Young's stress results from the deviation of the contact angle $\Theta$ from its static value $\Theta_{S}$. Such deviation drives the motion of the contact line in a way which, in the simplest case of a linear friction law, reads as follows:

$$
\begin{aligned}
D \gamma\left(\cos \Theta-\cos \Theta_{S}\right) & =U_{C L} & & \text { if } \Theta_{S}>0 \quad \text { (partial wetting), } \\
D \gamma(\cos \Theta-1) & =\max \left\{U_{C L}, 0\right\} & \text { if } \Theta_{S}=0 & \text { (complete wetting). }
\end{aligned}
$$

Here $U_{C L}$ is the speed of the contact line, $\gamma$ denotes the liquid-vapor surface tension, and $1 / D$ is an effective friction coefficient at the contact line. Note that the dynamic contact angle is strictly larger than the static one if the wet region expands, smaller (or equal, in complete wetting) if it contracts.

All together, (1.1) and (1.2) introduce two parameters in the problem, $B$ and $D$, which account for the effective friction at the liquid-solid and liquid-solid-vapor interfaces, respectively. The general goal of this note is to discuss the effect of these parameters on the evolution of a droplet, assumed for simplicity to be symmetric, which spreads over a horizontal substrate. To this aim, it is convenient to argue in the regime of lubrication approximation, which we introduce now.

1.2. Lubrication approximation and its dissipative structure. Lubrication approximation (see e.g. [21]) is a tool to reduce the complexity of the Navier-Stokes system while retaining the effects of both capillary forces and frictional forces (viscous friction in the bulk, surface friction at the liquid-solid interface, and contact-line friction at the liquid-solid-vapor interface). Lubrication approximation is based on a separation of the (macroscopic) lengthscales, which (in the presence of a contact line) has been rigorously justified in two model cases [13, 20]. Namely, the typical vertical lengthscale $Z$ is assumed to be much smaller than the typical horizontal lengthscale $X$, and the typical timescale is chosen so to retain the effects of both surface tension and viscosity:

$$
\varepsilon=\frac{Z}{X} \ll 1, \quad T=\frac{3 \mu}{\gamma} \frac{X^{4}}{Z^{3}} .
$$

Introducing new independent variables according to the above scaling,

$$
(t, x, z):=\left(\frac{\tau}{T}, \frac{\xi}{X}, \frac{\zeta}{Z}\right),
$$

and performing a careful asymptotic expansion in $\varepsilon$ (see e.g. [21, 7, 25]), one obtains a limiting evolution which consists in a fourth order free boundary problem for the normalized height of the liquid film, $h(t, x)$, and the extent of the wetted region, $(-s(t), s(t))$ (for simplicity, we assume $h$ to be symmetric with respect to $x=0$ ):

$$
\begin{cases}h_{t}+(h u)_{x}=0, u=\left(h^{2}+b h\right) h_{x x x} & \text { in }(0, s(t)), \\ h=0, \frac{\mathrm{d}}{\mathrm{d} t} s(t)=\lim _{x \rightarrow s(t)^{-}} u & \text { at } x=s(t), \\ h_{x}=h_{x x x}=0 & \text { at } x=0,\end{cases}
$$


and the free boundary condition (1.2) translates into

$$
d\left(h_{x}^{2}-\theta_{S}^{2}\right)=\left\{\begin{array}{ll}
\frac{\mathrm{d} s}{\mathrm{~d} t} & \text { if } \theta_{S}>0, \\
\max \left\{\frac{\mathrm{d} s}{\mathrm{~d} t}, 0\right\} & \text { if } \theta_{S}=0
\end{array} \quad \text { at } x=s(t) .\right.
$$

Here $u$ represents the normalized mean horizontal velocity of the liquid phase,

$$
b=\frac{3 \mu B}{Z}, \quad d=\frac{3 D \mu X}{2 Z}, \quad \text { and } \quad \theta_{S}=\frac{\Theta_{S}}{\varepsilon},
$$

where $\theta_{S}$ is a normalized static contact angle.

Now, it follows from a simple asymptotic expansion near $x=s(t)$ that the equation in (1.3) does not possess receding traveling waves with zero contact angle (see [4, 6] for the general structure of traveling waves for thin-film equations); in other words, $\frac{\mathrm{d} s}{\mathrm{~d} t} \geq 0$ whenever $h_{x}=0$ at the contact line. Therefore (1.4) simplifies to

$$
d\left(h_{x}^{2}-\theta_{S}^{2}\right)=\frac{\mathrm{d} s}{\mathrm{~d} t} \quad \text { at } \quad x=s(t)
$$

The free boundary problem (1.3)-(1.6) preserves the dissipative structure of the original system: as formally shown in [7], a sufficiently smooth solution to (1.3)-(1.6) satisfies

$$
\frac{\mathrm{d}}{\mathrm{d} t} \int_{0}^{s(t)} \frac{1}{2}\left(h_{x}^{2}+\theta_{S}^{2}\right) \mathrm{d} x=-\frac{1}{2 d}\left(\frac{\mathrm{d} s}{\mathrm{~d} t}\right)^{2}-\int_{0}^{s(t)} \frac{u^{2}}{h+b} \mathrm{~d} x .
$$

The integral on the left-hand side corresponds, to leading order in lubrication approximation, to the surface energy of the droplet, and accounts (via $\theta_{S}$ and the Young's law) for all three surface tension coefficients (liquid/solid, liquid/vapor, and solid/vapor) which enter into the system (see e.g. [3]). The two terms at the right-hand side of (1.7) encode the two different means of free energy dissipation: the latter, which is standard in this field, represents viscous friction both in the liquid's bulk and at the liquid/solid interface; the former instead represents friction at the contact line and is specific to the free boundary condition proposed in [23]. As expected, it vanishes when the effective friction coefficient $1 / d$ does.

1.3. Scaling laws without contact-line friction. For the ease of presentation, in what follows we assume (where not otherwise stated) that the droplet has unit mass,

$$
M=1
$$

(the general case can be easily recovered by scaling; see Section 3). In classical models, (1.6) is replaced by its frictionless counterpart, $1 / d=0$ :

$$
h_{x} \equiv-\theta_{S} \quad \text { at } \quad x=s(t),
$$

which amounts to assume an instantaneous enforcement of equilibrium at the contact line. In this case, the droplet's dynamics are known to be influenced only logarithmically by the slippage model, at least at intermediate timescales. This fact has been first observed by Hocking for $\theta_{S}>0$ (see also Cox [8] for the case of rough surfaces) by matched asymptotic methods. More precisely, in [17] a relation is obtained between the contact-line velocity and the macroscopic contact angle, $\theta_{M}$, defined there as the 
slope of the unique even arc of parabola having the same mass and support at its zero:

$$
p(s, x)=\frac{3}{4 s^{3}}\left(s^{2}-x^{2}\right)_{+}, \quad \theta_{M}=\left|\partial_{x} p(s, s)\right|=\frac{3}{2 s^{2}} .
$$

In the present two-dimensional case, it reads as follows:

$$
\theta_{M}^{3} \sim \theta_{S}^{3}+3 s^{\prime} \log \left(\frac{s \theta}{b}\right) .
$$

In the case $\theta_{S}=0$, the same logarithmic correction was obtained by Hocking in [18] and leads to the following scaling law for the speed of the contact line, which is often referred to as the logarithmic correction to Tanner's law [26]:

$$
s \sim\left(\frac{t}{\log \left(\frac{1}{b^{7} t}\right)}\right)^{1 / 7} .
$$

The scaling law (1.11) was then inferred in [2] by a different formal argument which used quasi-selfsimilar solutions, and rigorously derived in [12] for the boundary of the "macroscopic support", $(-a(t), a(t))=\{h(t, \cdot)>b\}$, i.e. replacing $s(t)$ by $a(t)$ in (1.9) and (1.11). In the latter two contributions, the time window of validity of (1.11) is also obtained:

$$
s_{0}^{7} \log \left(\frac{1}{b s_{0}}\right) \ll t \ll b^{-7} .
$$

Note that the appearance of an intermediate timescale is real: on one hand, it takes a certain time for the droplet to forget its initial shape; on the other hand, for large times $h \ll b$ on the whole support, hence the evolution is governed by slippage alone and $s$ will scale like $t^{1 / 6}$. Again in complete wetting, analogous logarithmic corrections were obtained by de Gennes [9] for a related model in which the contact angle condition is replaced by the action of van der Waals forces.

1.4. Scaling laws with contact-line friction. In the presence of contactline friction the situation is more complicated, since the scaling laws will depend not only on whether $\theta_{S}$ is zero or not, but also on the relation between the two parameters $b$ and $d$. In particular, due to the presence of two parameters, more than one intermediate scaling law should be expected in general. Indeed, in [25], formal considerations based on the dissipation relation (1.7) have been worked out in the complete wetting regime, $\Theta_{S}=0$. Three timescales are identified:

- an early stage, dominated by contact-line friction, where $s(t) \sim t^{1 / 5}$;

- a moderate stage, dominated by viscous friction, where $s(t) \sim t^{1 / 7}$;

- a final stage, dominated by surface friction, where $s(t) \sim t^{1 / 6}$.

Such behavior has been validated by numerical simulations of (1.3)-(1.6). The goal of this contribution is to give a more precise and more quantitative description of these scaling laws, in the spirit of (1.10), (1.11), and (1.12), covering also the case of partial wetting (see Section 2.4). As a by-product, we will obtain a matched asymptotic expansion of solutions to (1.3)-(1.6) for a wide class of free boundary conditions relating the speed and the contact angle.

We point out that our analysis is confined to the case in which lubrication approximation is valid in the entire film, including the contact line region. This may 
not be true due to various circumstances. Among them is the case of a static contact angle $\Theta_{S}$ which is not "small" (in the sense of (1.5)). In this case our analysis is not applicable and a different argument has to be used in order to infer the scaling laws. The numerical results in [23, Section VII] suggest that long-time relations of the form (1.10) will remain valid. We should also point out that our analysis neglects the effect of disjoining/conjoining pressure, which adds a surface potential term to the expression for $u$ in (1.3); we refer to [25] both for the details and for related scaling laws in complete wetting.

\section{Results and outline}

In Section 3 we rewrite (1.3)-(1.6) in dimensionless form. This highlights the crucial role of the parameter

$$
k=\frac{d M}{b^{2}},
$$

which may be seen as a measure of the relative strength of surface friction versus contact-line friction. In summarizing the results of our analysis, we assume once again that $M=1$ and we disregard universal constants.

2.1. Scaling laws in complete wetting. If $\theta_{S}=0$, we will argue that

(A) for a stronger contact-line friction, $d \lesssim b^{2}$, the droplet displays an early timescale dominated by contact-line friction and a final timescale dominated by surface friction:

$$
s \sim\left\{\begin{array}{l}
(d t)^{1 / 5} \text { if } \frac{s_{0}^{5}}{d} \ll t \ll \frac{b^{5}}{d^{6}} \quad\left(\text { and } s_{0} \ll \frac{b}{d}\right), \\
(b t)^{1 / 6} \text { if } t \gg \frac{b^{5}}{d^{6}} ;
\end{array}\right.
$$

(B) for a stronger surface friction, $b^{2} \ll d$, the droplet displays an early timescale dominated by contact-line friction, a moderate timescale dominated by viscous friction, and a final timescale dominated by surface friction:

$$
s \sim\left\{\begin{array}{ll}
(d t)^{1 / 5} & \text { if } \frac{s_{0}^{5}}{d} \ll t \ll \frac{1}{d^{7 / 2} \log ^{5 / 2} \frac{d}{b^{2}}}
\end{array} \text { (and } s_{0}^{2} \ll \frac{1}{d \log \frac{d}{b^{2}}}\right),
$$

The scaling laws in (B) quantify those predicted in [25]. A main difference may be noted:

- for a stronger contact-line friction, case (A), the system bypasses the moderate timescale dominated by viscous friction.

One also notices that

- for a stronger surface friction, case (B), the moderate regime is logarithmically corrected by surface friction, as in the case of zero contact-line friction (see (1.11)-(1.12));

- all timescales, besides the final one, depend both on surface and on contactline friction.

As already pointed out in Section 1.3, the lower bounds on the initial times are real: they correspond to the time that the system needs to "forget" its initial shape and to relax to a quasi-static configuration. 
2.2. A heuristic argument and its limitation. In Section 4 we look at the case of complete wetting, $\theta_{S}=0$, and we present a variant of a simple heuristic argument, based on the dissipation relation (1.7), which has already been used in this framework (see the discussion in Section 4). Though this argument is already capable to predict (2.1) and (2.2), it relies on quite a heavy hypothesis: the quasistatic equilibrium configuration of $h$ (see (1.9) and (4.1) below) must be postulated up to the contact-line. This assumption may not be true in general; for instance, it is certainly false in the case of a null contact-line friction, $1 / d=0$, since the condition $h_{x}=0$, as given by (1.8), is incompatible with (1.9). In Remark 4.1 we argue that the heuristic argument lacks self-consistency (in the sense that the conclusions are incompatible with that assumption) when the evolution is not driven by contact-line friction. This points to the necessity of working out a matched asymptotic study of (1.3)-(1.6) which avoids such a strong postulate.

2.3. Matched asymptotic analysis. After the works of Hocking $[17,18]$ and of Cox [8], matched asymptotic with speed-dependent contact angle conditions have been extensively performed in the past $[15,11,16]$. However, none of them includes (1.6), and the scaling assumptions used are not always sharp or easy to reconstruct. Hence, here we extend, modify, and simplify the asymptotic in a way which includes (1.6) and keeps track of all the assumptions used. Up to the extent we need for (1.6), we may argue for a rather general relation between speed and contact angle,

$$
\left|h_{x}(t, s(t))\right|=\theta=\theta\left(s^{\prime}(t), \theta_{S}\right), \quad \theta \gtreqless \theta_{S} \quad \text { for } \quad s^{\prime} \gtreqless 0,
$$

which makes the results potentially applicable to different boundary conditions and therefore, we believe, of independent interest. The asymptotic is based on the assumptions that the evolution is "slow" and quasi-static, and yields the following: if

$$
0 \leq s^{6} s^{\prime} \ll 1 \text { and } \quad b s \ll 1,
$$

then

$$
\theta_{M}^{3} \sim \begin{cases}\theta^{3}+3 s^{\prime} \log \left(\frac{s \theta}{b}\right) & \text { if } b \ll s \theta \quad \text { and } \quad s^{\prime} \ll \theta^{3} \\ 3 s^{\prime} \log \left(\frac{s\left(s^{\prime}\right)^{1 / 3}}{b}\right) & \text { if } b^{3} \ll s^{3} s^{\prime} \quad \text { and } \quad s^{\prime} \gg \theta^{3},\end{cases}
$$

where $\theta_{M}$ is defined as in (1.9). The first assumption in (2.4) says that the droplet spreads and spreads slowly; in particular, it rules out of the analysis an initial timescale during which the evolution is governed by the droplet's initial shape. The second one ensures (via mass conservation) that $h(t, \cdot) \gg b$ on most of its support, which motivates calling $\theta_{M}$ a macroscopic contact angle. Of course, (2.5) recovers the earlier results in $[17,18]$ when $\theta \equiv \theta_{S}$. In Section 5 we also obtain an asymptotic relation between $s$ and $\theta$, valid when $h \ll b$ but the evolution is "slow" and quasi-static:

$$
\left(\frac{3}{2 s^{2}}\right)^{3} \sim \theta^{3} \quad \text { if } b s \gg 1, s^{5} s^{\prime} \ll b, \text { and } \theta>0 .
$$

In Section 6 and Section 7 we consider the specific contact-angle condition (1.6) in the regime of complete wetting, and we use ODE arguments to pass from (2.5) and (2.6) to the early and moderate scaling laws in (2.1) and (2.2). In the particular case $1 / d=0,(1.11)-(1.12)$ are also recovered. The scaling laws for long time are obtained in Section 8 by a different asymptotic which assumes a quasi-selfsimilar profile of the 
solution. As a consequence, one may conclude that $\theta_{M}$ and $\theta$ are indeed "relatively close" to each other, which a-posteriori justifies the heuristic argument described in Section 2.2 .

2.4. Scaling laws in partial wetting. In the case of partial wetting, $\theta_{S}>0$, the profile of a spreading droplet converges (exponentially, see Section 9) to the unique steady state with given mass and contact angle $\theta_{S}$ as $t \rightarrow+\infty$ : assuming $M=1$,

$$
h \rightarrow \frac{3}{4 s_{\infty}^{3}}\left(s_{\infty}^{2}-x^{2}\right)_{+} \quad \text { and } \quad s \uparrow s_{\infty}=\sqrt{\frac{3}{2 \theta_{S}}} \quad \text { as } t \rightarrow+\infty .
$$

We focus on the most interesting case of

$$
\theta_{S} \gg b^{2}, \text { i.e. } b s_{\infty} \ll 1,
$$

which guarantees the persistence for all times of a macroscopic profile. In Section 9 we argue that, for sufficiently large times, the system evolves in accordance with the Cox-Hocking relation (1.10) between the effective and the microscopic contact angle. Hence, also in partial wetting the contact-line friction plays no role for large times. However, it turns out that there are still intermediate timescales which are influenced by contact-line friction. We illustrate the results in words for $M=1$, neglecting a (logarithmically short) transition timescale (the reader is referred to Section 9 for the precise statements):

(i) if $d \ll \theta_{S}$, then (1.10) is preceded by an early timescale dominated by contactline friction;

(ii) if $\theta_{S} \ll d$, then (1.10) is preceded by an early timescale dominated by contactline friction and a moderate timescale dominated by viscous friction.

These results identify the ratio $d / \theta_{S}$ as the threshold parameter in the partial wetting regime. In addition, the upper bounds on the timescales permit one to quantify the time in which (1.10) takes over; again up to a logarithmic correction, the analysis in Section 9 shows that

$$
(1.10) \Longleftrightarrow t \gg \begin{cases}\frac{1}{d \theta_{S}^{5 / 2}} 1 & \text { if } d \ll \theta_{S}, \\ \frac{\theta_{S}^{7 / 2} \log ^{1 / 6}\left(\frac{\theta_{S}}{b^{2}}\right)}{\text { if }} & \theta_{S} \ll d .\end{cases}
$$

\section{Dimensionless form}

It is convenient to scale all quantities in such a way that the mass is 1 and the equation is parameter-free:

$$
x=\frac{M}{b} \hat{x} \quad \text { and } \quad s=\frac{M}{b} \hat{s}, \quad h=b \hat{h}, \quad t=\frac{M^{4}}{b^{7}} \hat{t} .
$$

In particular, the nonlinearity $m(h)=h^{3}+b h^{2}$ turns into $m(\hat{h})=\hat{h}^{3}+\hat{h}^{2}$ : the transition between the two regimes of $m, h \sim b$, in the new variables occurs at $\hat{h} \sim 1$. The free boundary condition (1.6) reads as

$$
\frac{d M}{b^{2}}\left(\hat{h}_{\hat{x}}^{2}-\frac{M^{2}}{b^{4}} \theta_{S}^{2}\right)=\frac{\mathrm{d} \hat{s}}{\mathrm{~d} \hat{t}} \quad \text { at } \hat{x}=\hat{s}(\hat{t}) .
$$

Hence, introducing the parameters

$$
\alpha_{S}=\frac{M}{b^{2}} \theta_{S}, \quad k=\frac{d M}{b^{2}}
$$


and removing all hats, (1.3)-(1.6) read as

$$
\begin{cases}h_{t}+(h u)_{x}=0, u=\left(h^{2}+h\right) h_{x x x}, h>0 & \text { in }(0, s(t)), \\ h_{x}=h_{x x x}=0 & \text { at } x=0, \\ h=0, s^{\prime}(t)=\lim _{x \rightarrow s(t)^{-}} u(t, x)=k\left(h_{x}^{2}-\alpha_{S}^{2}\right) & \text { at } x=s(t),\end{cases}
$$

and the dissipation relation (1.7) transforms into

$$
\frac{\mathrm{d}}{\mathrm{d} t} \int_{0}^{s(t)} \frac{1}{2}\left(h_{x}^{2}+\alpha_{S}^{2}\right) \mathrm{d} x=-\frac{1}{2 k}\left(s^{\prime}(t)\right)^{2}-\int_{0}^{s(t)} \frac{u^{2}}{h+1} \mathrm{~d} x .
$$

\section{A heuristic argument and its limitation}

In this section we consider the case of complete wetting:

$$
\alpha_{S}=0 .
$$

In the case of null contact-line friction, $1 / d=0$, the scaling law (1.11) was first observed by Hocking [18] and then rigorously derived in [12] for the boundary $a(t)$ of the "macroscopic support", $(-a(t), a(t))=\{h(t, \cdot)>b\}$. While Hocking uses careful matched asymptotic expansions, the heuristic behind the rigorous results in [12] is much simpler: it relies on the energy dissipation mechanism encoded by (3.2) and is inspired by that used by de Gennes in [9]; more recently, Glasner [14] has given a detailed interpretation to this heuristic in terms of gradient flows. The heuristic in [12] is based on assuming that the evolution is quasi-static on the macroscopic support and that most of the energy is contained and dissipated there (though near its boundary): this allows one to obtain an ODE for a single unknown, $a(t)$, thus avoiding all the subtleties of "matching" with a microscopic region near the contact line.

If instead $1 / d>0,(3.2)$ contains a term which acts at the contact line, and which therefore already contains the unknown $s^{\prime}(t)$ (or equivalently, $\left.h_{x}\right|_{x=s(t)}$ ). Hence, in revisiting the aforementioned heuristic, in order to get a closed ODE one is forced to use $s(t)$ instead of $a(t)$ as the unknown, i.e. to argue in the whole support $(-s(t), s(t))$ rather than just on the macroscopic one. In particular, one has to assume that the evolution is quasi-static in the whole support, in the sense that the droplet's profile is, at leading order, in equilibrium given its mass and its whole support:

$$
h \sim \frac{1}{s^{3}}\left(s^{2}-x^{2}\right) \quad \text { for } x \in(-s, s)
$$

(here and after we disregard universal constants). Then, by a simple computation,

$$
\frac{\mathrm{d}}{\mathrm{d} t} \int_{0}^{s} h_{x}^{2} \mathrm{~d} x \sim-\frac{s^{\prime}}{s^{4}}
$$

In order to compute the rate of dissipation in $(0, s(t))$, we pick the simplest possible form of the velocity field $u$ such that $u=0$ at $x=0$ and $u=s^{\prime}(t)$ at $x=s(t)$ :

$$
u \sim \frac{x s^{\prime}}{s} \text {. }
$$

Then

$$
\int_{0}^{s} \frac{u^{2}}{h+1} \mathrm{~d} x \sim \frac{s^{\prime 2}}{s^{2}}\left(\int_{\{h \geq 1\}} \frac{x^{2}}{h} \mathrm{~d} x+\int_{\{h \leq 1\}} x^{2} \mathrm{~d} x\right) .
$$


In view of (4.1), the first integral on the right-hand side is zero if $s \gg 1$. Simple computations using (4.1) then yield

$$
\int_{0}^{s} \frac{u^{2}}{h+1} \mathrm{~d} x \sim \begin{cases}s^{2}\left(s^{\prime}\right)^{2} \log \frac{1}{s} & \text { if } s \ll 1, \\ s\left(s^{\prime}\right)^{2} & \text { if } s \gg 1 .\end{cases}
$$

Plugging (4.1) and (4.2) into (3.2) we obtain

$$
-\frac{s^{\prime}}{s^{4}} \sim \begin{cases}-\frac{\left(s^{\prime}\right)^{2}}{k}-s^{2}\left(s^{\prime}\right)^{2} \log \frac{1}{s} & \text { if } s \ll 1, \\ -\frac{\left(s^{\prime}\right)^{2}}{k}-s\left(s^{\prime}\right)^{2} & \text { if } s \gg 1,\end{cases}
$$

that is,

$$
\frac{1}{s^{\prime}} \sim \begin{cases}\frac{s^{4}}{k}+s^{6} \log \frac{1}{s} & \text { if } s \ll 1, \\ \frac{s^{4}}{k}+s^{5} & \text { if } s \gg 1 .\end{cases}
$$

We note that

$$
\begin{aligned}
\frac{s^{4}}{k} \gg s^{6} \log \frac{1}{s} & \Longleftrightarrow \frac{1}{k} \gg s^{2} \log \frac{1}{s^{2}}, \\
\frac{s^{4}}{k} \gg s^{5} & \Longleftrightarrow \frac{1}{k} \gg s .
\end{aligned}
$$

Hence we must distinguish two cases.

(1). If $k \ll 1$, then (4.4) is always satisfied for $s \ll 1$, and (4.3) reads as

$$
\frac{1}{s^{\prime}} \sim\left\{\begin{aligned}
\frac{s^{4}}{k} & \text { if } s \ll \frac{1}{k}, \\
s^{5} & \text { if } s \gg \frac{1}{k} .
\end{aligned}\right.
$$

We assume that $s_{0} \ll 1 / k$, so that both the regimes in (4.6) are seen. Then, solving (4.6) renders

$$
s \sim \begin{cases}s_{0}+(k t)^{1 / 5} \sim(k t)^{1 / 5} & \text { if } \frac{s_{0}^{5}}{k} \ll t \ll \frac{1}{k^{6}}, \\ t^{1 / 6} & \text { if } t \gg \frac{1}{k^{6}} .\end{cases}
$$

(2). If $k \gg 1$, then (4.5) is never satisfied for $s \gg 1$, whereas for $s \ll 1$ (4.4) may be inverted as follows:

$$
(4.4) \Longleftrightarrow \frac{1}{k \log k} \gg s^{2} \text {. }
$$

Therefore (4.3) reads as

$$
\frac{1}{s^{\prime}} \sim \begin{cases}\frac{s^{4}}{k} & \text { if } s^{2} \ll \frac{1}{k \log k}, \\ s^{6} \log \frac{1}{s} & \text { if } \frac{1}{k \log k} \ll s^{2} \ll 1, \\ s^{5} & \text { if } s \gg 1\end{cases}
$$

Assuming that $s_{0}^{2} \ll \frac{1}{k \log k}$ and solving this ODE (see Section 7.2 for details) yields

$$
s \sim \begin{cases}s_{0}+(k t)^{1 / 5} \sim(k t)^{1 / 5} & \text { if } \frac{s_{0}^{5}}{k} \ll t \ll \frac{1}{k^{7 / 2} \log ^{5 / 2} k}, \\ \left(\frac{t}{\log \frac{1}{t}}\right)^{1 / 7} & \text { if } \frac{1}{k^{7 / 2} \log ^{5 / 2} k} \ll t \ll 1, \\ t^{1 / 6} & \text { if } t \gg 1 .\end{cases}
$$


Returning to the original variables, (4.7) and (4.9) coincide with (2.1) and (2.2), respectively.

REMARK 4.1. It must be pointed out that assuming (4.1) implicitly postulates that

$$
\left.h_{x}\right|_{x=s(t)} \stackrel{(4.1)}{\sim}-\frac{1}{s^{2}}
$$

Indeed, one can easily check that the equivalent formulation of (3.2) (with $\alpha_{S}=0$ ),

$$
\frac{\mathrm{d}}{\mathrm{d} t} \int_{0}^{s(t)} \frac{1}{2} h_{x}^{2} \mathrm{~d} x \stackrel{(3.2),(3.1)}{=}-\left.\frac{k}{2} h_{x}^{4}\right|_{x=s(t)}-\int_{0}^{s(t)} \frac{u^{2}}{h+1} \mathrm{~d} x
$$

with the contact-angle given by (4.10), leads exactly to the same asymptotic, (4.6) and (4.8). On the other hand, rewriting (4.10) in the form

$$
\left.s^{\prime}(t) \stackrel{(3.1)}{=} k h_{x}^{2}\right|_{x=s(t)} \stackrel{(4.10)}{\sim} \frac{k}{s^{4}(t)}
$$

and comparing it with (4.6) and (4.8), one immediately sees that the heuristic argument is self-consistent only along those time-scales in which $s^{\prime}$ depends on $k$.

Remark 4.1 points to the need for a more careful asymptotic analysis which avoids postulating (4.1). We will work it out in the rest of this paper.

\section{Matched asymptotic and the macroscopic contact angle}

We work under the more general boundary condition (2.3), which in the dimensionless form of Section 3 reads as

$$
\left|h_{x}(t, s(t))\right|=\alpha\left(s^{\prime}(t), \alpha_{S}\right), \quad \alpha\left(s^{\prime}, \alpha_{S}\right) \gtreqless \alpha_{S} \text { for } \quad s^{\prime} \gtreqless 0 .
$$

Note that the contact-angle condition in (3.1) is included in (5.1) by letting

$$
\alpha\left(s^{\prime}, \alpha_{S}\right)=\sqrt{\frac{s^{\prime}}{k}+\alpha_{S}^{2}} .
$$

The asymptotic is based on two main assumptions:

(I) the evolution within the liquid's bulk is "quasi-static";

(II) the evolution within the liquid's bulk is "slow".

The former is of a qualitative nature. In order to make it more precise, it is convenient to introduce a variable transformation which differs from those used in earlier studies and yields sharp scaling assumptions. It fixes the free boundary and preserves mass:

$$
h(t, x)=\frac{1}{s(t)} H(t, y), \quad y=\frac{x}{s(t)} \in(0,1) .
$$

Then

$$
s^{6} s^{\prime}(y H)_{y}-s^{7} H_{t}=\left(\left(H^{3}+s H^{2}\right) H_{y y y}\right)_{y} \quad \text { in } \quad(0, \infty) \times(0,1) .
$$

A quasi-static evolution of the liquid's bulk means that, except maybe for a region where $H \ll 1, H$ depends on time only through the modulations given by $s$ and $s^{\prime}$. Hence (5.3) reads as

$$
\left(s^{6} s^{\prime}\right)(y H)_{y} \sim\left(\left(H^{3}+s H^{2}\right) H_{y y y}\right)_{y},
$$


which may be integrated once with respect to $y$ (from $y=0$ ), obtaining

$$
\left(s^{6} s^{\prime}\right) y H \sim\left(H^{3}+s H^{2}\right) H_{y y y} \quad \text { in }(0,1) .
$$

We now think of $H$ and its derivatives to be $O(1)$; then (5.4) shows a scaling-wise natural way to quantify the notion of a "slow" evolution within the liquid's bulk:

$$
s^{6} s^{\prime} \ll 1 \text { if } s \ll 1
$$

and

$$
s^{5} s^{\prime} \ll 1 \text { if } s \gg 1
$$

Note that four conditions are to be imposed for $H$, whereas (5.4) is of third order; we'll use

$$
\left.H_{y}\right|_{y=0}=0,\left.\quad H\right|_{y=1}=0, \quad \int_{0}^{s(t)} H(s(t), x) \mathrm{d} x=\frac{1}{2}
$$

to determine $H$, and

$$
\left.H_{y}\right|_{y=1}=-\alpha s^{2}
$$

to determine a relation between $s$ and $s^{\prime}$. Provided (5.5) holds, we obtain the following asymptotic:

$$
\left(\frac{3}{2 s^{2}}\right)^{3} \sim \begin{cases}\alpha^{3}+3 s^{\prime} \log (s \alpha) & \text { if } 1 \ll s \alpha \text { and } s^{\prime} \ll \alpha^{3} \\ 3 s^{\prime} \log \left(s\left(s^{\prime}\right)^{1 / 3}\right) & \text { if } 1 \ll s^{3} s^{\prime} \text { and } s^{\prime} \gg \alpha^{3} .\end{cases}
$$

If instead (5.6) holds, then

$$
\left(\frac{3}{2 s^{2}}\right)^{3} \sim \alpha^{3} \quad \text { if } \alpha>0
$$

Returning to the original variables we obtain (2.5) and (2.6). In the rest of the section we provide the details for both. The first one is by far less obvious.

5.1. Slow evolution with a macroscopic profile: the outer expansion.

We first consider the case $s \ll 1$, which in view of mass conservation implies that $H \gg 1$ in the liquid's bulk, i.e., a macroscopic profile exists. Since $s \ll 1,(5.4)$ and (5.8) simplify to

$$
\left(s^{6} s^{\prime}\right) y H \sim H^{3} H_{y y y} \quad \text { in } \quad(0,1)
$$

and

$$
\left.H_{y}\right|_{y=1}=0,
$$

respectively. In view of (5.5), we expand $H$ in powers of $s^{6} s^{\prime}$ :

$$
H=H_{0}(y)+\left(s^{6} s^{\prime}\right) H_{1}(y)+\text { lower order terms. }
$$

At zeroth order, (5.11) and (5.7) read as

$$
\left\{\begin{array}{l}
\left(H_{0}\right)_{y y y}=0 \text { in }(0,1) \\
\left.\left(H_{0}\right)_{y}\right|_{y=0}=0,\left.H_{0}\right|_{y=1}=0, \int_{0}^{1} H_{0}(y) \mathrm{d} y=\frac{1}{2}
\end{array}\right.
$$


A simple calculation shows that the solution of (5.13) is

$$
H_{0}(y)=\frac{3}{4}\left(1-y^{2}\right)
$$

Since the contact-angle condition (5.12) can not be satisfied, we proceed to first order. For $H_{1}$, we obtain

$$
\left\{\begin{array}{l}
\left(H_{1}\right)_{y y y}=\frac{y}{H_{0}^{2}}=\frac{16 y}{9\left(1-y^{2}\right)^{2}} \quad \text { in }(0,1), \\
\left.\left(H_{1}\right)_{y}\right|_{y=0},\left.H_{1}\right|_{y=1}=0, \int_{0}^{1} H_{1}(y) \mathrm{d} y=0
\end{array}\right.
$$

Three integrations yield, after lengthy but straightforward computations,

$$
H_{1}(y)=\frac{8-9 B}{18}\left(1-y^{2}\right)+\frac{4}{9}((1+y) \log (1+y)+(1-y) \log (1-y)-2 \log 2),
$$

where $B=\left(H_{1}\right)_{y y}(0)$ must be determined via the mass constraint. After an additional calculus exercise, one sees that $B=-4 / 9$, and therefore

$$
\begin{aligned}
H_{y} & \sim\left(H_{0}+s^{6} s^{\prime} H_{1}\right)_{y}=-\frac{3}{2} y+s^{6} s^{\prime}\left(-\frac{4}{3} y+\frac{4}{9} \log \left(\frac{1+y}{1-y}\right)\right) \\
& \sim-\frac{3}{2}+\frac{4}{9} s^{6} s^{\prime} \log \left(\frac{1}{1-y}\right) \quad \text { as } y \rightarrow 1 .
\end{aligned}
$$

Since $H_{y}$ has a logarithmic singularity as $y \rightarrow 1$, we can not impose the contact-angle condition (5.12). This points to the necessity of an inner expansion which permits to cancel the singularity by a suitable matching. Before proceeding we observe that, in terms of the original variables, (5.14) reads as

$$
h_{x} \sim-\frac{3}{2 s^{2}}+\frac{4}{9} s^{4} s^{\prime} \log \left(\frac{s}{s-x}\right) \text { for } \frac{s}{s-x} \gg 1 .
$$

5.2. Slow evolution with a macroscopic profile: the inner expansion. Near the free boundary we follow $[17,18]$ and use the scaling of a traveling wave,

$$
h(t, x)=f(\xi), \quad \xi=s(t)-x .
$$

We impose the touchdown condition, $f(0)=0$, the contact angle condition, $f_{\xi}=\alpha$ at $\xi=0$, and the kinematic condition, $f=f u=0$ at $\xi=0$. Then, after one integration, we see that for each $t>0$

$$
\begin{cases}f_{\xi \xi \xi}=-\frac{s^{\prime}}{f^{2}+f} & \text { for } \xi>0, \\ f=0 & \text { at } \xi=0, \\ f_{\xi}=\alpha & \text { at } \xi=0 .\end{cases}
$$

In order to achieve a matching with the solution in the outer region, $f_{\xi}$ must be no more than logarithmically large at infinity. This singles out the unique solution of (5.16) such that $f_{\xi \xi} \rightarrow 0$ as $\xi \rightarrow+\infty$, as given by Theorem 1.1 in [7]. A simple asymptotic expansion of (5.16) shows that this solution is such that

$$
f(\xi) \sim\left(3 s^{\prime}\right)^{1 / 3} \xi(\log \xi)^{1 / 3} \quad \text { as } \xi \rightarrow+\infty \quad \text { if } \quad s^{\prime}>0 .
$$


In order to infer the asymptotic form of $f_{\xi}$ up to order 0 in $\xi$, we distinguish two regimes:

(1). $\beta=\frac{s^{\prime}}{\alpha^{3}} \ll 1$. In this case we rescale (5.16) according to $\hat{\xi}=\alpha \xi$, so that

$$
f_{\hat{\xi} \hat{\xi} \hat{\xi}}=-\frac{\beta}{f^{2}+f}, \quad f_{\hat{\xi}}(0)=1,
$$

and we linearize around $\beta=0: f=f_{0}+\beta f_{1}+\ldots$. At leading order in $\beta$ we have

$$
f_{0}=\hat{\xi} .
$$

At first order in $\beta$ we have

$$
\left(f_{1}\right)_{\hat{\xi} \hat{\xi} \hat{\xi}}=-\frac{1}{\hat{\xi}^{2}+\hat{\xi}} \quad \text { for } \xi>0, \quad f_{1}(0)=\left(f_{1}\right)_{\hat{\xi}}(0)=0 .
$$

After two integrations (using the boundary conditions), we obtain

$$
\begin{aligned}
\left(f_{1}\right)_{\hat{\xi}} & =(1+\hat{\xi}) \log (1+\hat{\xi})-\hat{\xi} \log \hat{\xi}=(1+\hat{\xi})\left(\log \hat{\xi}+\log \left(1+\frac{1}{\hat{\xi}}\right)\right)-\hat{\xi} \log \hat{\xi} \\
& \sim 1+\log \hat{\xi} \quad \text { as } \hat{\xi} \rightarrow+\infty
\end{aligned}
$$

Recombining (5.18) and (5.19), we see that

$$
f_{\hat{\xi}} \sim\left(f_{0}+\beta f_{1}\right)_{\xi} \sim 1+\beta(1+\log \hat{\xi}) \quad \text { for } \hat{\xi} \gg 1 .
$$

Recalling that $\beta \ll 1$, in terms of the outer variable the previous expression reads as follows:

$$
-h_{x} \sim \alpha+\frac{s^{\prime}}{\alpha^{2}} \log (\alpha(s-x)) \quad \text { for } \quad \alpha(s-x) \gg 1 .
$$

(2). $\beta=\frac{s^{\prime}}{\alpha^{3}} \gg 1$. In this case we scale (5.16) according to $\hat{\xi}=\left(s^{\prime}\right)^{1 / 3} \xi$, so that

$$
f_{\hat{\xi} \hat{\xi} \hat{\xi}}=-\frac{1}{f^{2}+f}, \quad f_{\hat{\xi}}(0)=\frac{1}{\beta^{1 / 3}} .
$$

At leading order in $\beta^{-1 / 3}$ we obtain that

$$
\left\{\begin{array}{l}
f_{\hat{\xi} \hat{\xi} \hat{\xi}}=-\frac{1}{f^{2}+f} \quad \text { in } \quad(0,+\infty), \\
f(0)=f_{\hat{\xi}}(0)=0, \quad \lim _{\hat{\xi} \rightarrow+\infty} f_{\hat{\xi} \hat{\xi}}(\hat{\xi})=0 .
\end{array}\right.
$$

Theorem 1.1 in [7] guarantees that (5.21) has a unique solution, and the asymptotic in (5.17) yields

$$
f_{\hat{\xi}} \sim \log ^{1 / 3}\left(\hat{\xi}^{3}\right) \quad \text { as } \hat{\xi} \rightarrow+\infty .
$$

In terms of the outer variables, this means that

$$
-h_{x} \sim\left(s^{\prime} \log \left(s^{\prime}(s-x)^{3}\right)\right)^{1 / 3} \quad \text { for } \quad s^{\prime}(s-x)^{3} \gg 1 .
$$


5.3. Slow evolution with a macroscopic profile: the matching. In the outer region, where $h \gg 1$, the velocity field $u=\left(h^{2}+h\right) h_{x x x} \sim h^{2} h_{x x x}$ has the same scaling of $h_{x}^{3}$. Therefore, in order to get a relation between the velocity and the macroscopic contact angle, it is natural to cube the expressions obtained for $h_{x}$. For the outer profile, at order one in $s^{6} s^{\prime}$ we find from (5.15) that

$$
h_{x}^{3} \sim-\left(\frac{3}{2 s^{2}}\right)^{3}+3 s^{\prime} \log \left(\frac{s}{s-x}\right) \quad \text { for } \quad(s-x) \ll s .
$$

For the inner profile, (5.20) (at order one in $s^{\prime} / \alpha^{3}$ ) and (5.22) yield

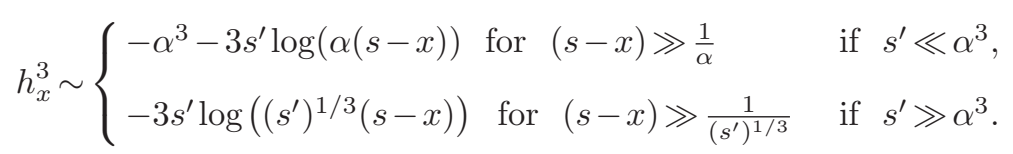

Having carefully tracked the scaling assumptions both in the outer and in the inner region allows one to simplify the matching with respect to $[17,18]$. Indeed, we just have to notice that the range of validity of the expansions (5.23) and (5.24) overlap if $1 \ll s \alpha$ when $s^{\prime} \ll \alpha^{3}$, and if $s^{3} s^{\prime} \gg 1$ when $s^{\prime} \gg \alpha^{3}$. In these cases we may equate them, and after a cancellation of the $\log (s-x)$ terms we obtain (5.9).

5.4. Slow evolution without macroscopic profile. Since $s \gg 1, H^{3}+$ $s H^{2} \sim s H^{2}$, so that (5.4) takes the form

$$
\left(s^{5} s^{\prime}\right) y \sim H H_{y y y} .
$$

Because of (5.6), we expand $H$ in powers of $s^{5} s^{\prime}: H=H_{0}+\left(s^{5} s^{\prime}\right) H_{1}+$ lower order terms. At zeroth order, as in Section 5.1, we recover

$$
H_{0}(y)=\frac{3}{4}\left(1-y^{2}\right)
$$

This solution meets the boundary condition $\left(H_{0}\right)_{y}=-\alpha s^{2}$ provided $\alpha>0$, and in terms of the original variables we obtain (5.10).

6. Intermediate scaling law in complete wetting without contact-line friction

As a first example, which we shall anyway need later on, we recover the wellknown logarithmic correction to Tanner's law stated in (1.11)-(1.12) in the case that $\alpha \equiv 0$. We will neglect universal constants.

Since $\alpha \equiv 0$, only the second regime in (5.9) is relevant. Hence, if

$$
s \ll 1,
$$

and if

$$
s^{6} s^{\prime} \ll 1, s^{3} s^{\prime} \gg 1
$$

then

$$
\frac{1}{s^{6}} \sim s^{\prime} \log \left(s^{3} s^{\prime}\right) .
$$

We now analyze (6.1)-(6.3) in the $\left(s, s^{\prime}\right)$ plane. First of all, we make (6.3) explicit (in what follows we shall often use this type of argument; we provide its details here once for all):

$$
\frac{1}{s^{6}} \sim s^{\prime} \log \left(s^{3} s^{\prime}\right) \Longleftrightarrow \frac{1}{s^{3}} \sim s^{3} s^{\prime} \log \left(s^{3} s^{\prime}\right) \stackrel{(6.2)}{\gg} 1
$$




$$
\begin{aligned}
& \Longleftrightarrow \frac{1}{s^{3} \log \left(\frac{1}{s^{3}}\right)} \sim s^{3} s^{\prime} \\
& \Longleftrightarrow s^{\prime} \sim \frac{1}{s^{6} \log \left(\frac{1}{s}\right)} .
\end{aligned}
$$

Then we observe that

$$
\begin{aligned}
& s^{6} s^{\prime} \ll 1 \stackrel{(6.4)}{\Longleftrightarrow} \frac{1}{\log \left(\frac{1}{s}\right)} \ll 1 \Longleftrightarrow s \ll 1, \\
& 1 \ll s^{3} s^{\prime} \stackrel{(6.4)}{\Longleftrightarrow} s^{3} \log \left(\frac{1}{s}\right) \ll 1 \Longleftrightarrow s \ll 1 .
\end{aligned}
$$

Hence (6.1)-(6.3) are equivalent to (6.1) and (6.4). If (6.1) is initially true, i.e. $s_{0}:=$ $s(0) \ll 1$, we may integrate (6.4): since

$$
\left(s^{7} \log \left(\frac{1}{s}\right)\right)^{\prime} \stackrel{(6.1)}{\sim} s^{6} \log \left(\frac{1}{s}\right) s^{\prime},
$$

we obtain

$$
s^{7} \log \left(\frac{1}{s}\right) \sim t \quad \text { provided } s_{0}^{7} \log \left(\frac{1}{s_{0}}\right) \ll t .
$$

We now check for how long (6.1) remains true:

$$
s \ll 1 \Longleftrightarrow s^{7} \ll 1 \Longleftrightarrow \frac{t}{\log \left(\frac{1}{t}\right)} \ll 1 \Longleftrightarrow t \ll 1,
$$

and in this case (6.5) may be inverted as before, yielding

$$
s^{7} \sim \frac{t}{\log \left(\frac{1}{t}\right)} \text { provided } s_{0}^{7} \log \left(\frac{1}{s_{0}}\right) \ll t \ll 1 \text { and } s_{0} \ll 1 .
$$

Note that the time window is not empty since $s_{0} \ll 1$. Returning to the original variables we recover (1.11)-(1.12). Large timescales will be analyzed in Section 8.

7. Intermediate scaling laws in complete wetting with contact-line friction

We now focus on the specific boundary condition proposed in [23] in the case of complete wetting, $\alpha_{S}=0$. In view of (5.2), we then have

$$
\alpha=\sqrt{s^{\prime} / k} .
$$

We will neglect universal constants, and argue that:

(I) if $k \lesssim 1$ and $s_{0} \ll \frac{1}{k}$, then

$$
s(t) \sim(k t)^{1 / 5} \quad \text { if } \quad \frac{s_{0}^{5}}{k} \ll t \ll \frac{1}{k^{6}} ;
$$

(II) if $k \gg 1$ and $s_{0}^{2} \ll \frac{1}{k \log k}$, then

$$
s(t) \sim \begin{cases}(k t)^{1 / 5} & \text { if } \frac{s_{0}^{5}}{k} \ll t \ll \frac{1}{k^{7 / 2} \log ^{5 / 2} k}, \\ \left(\frac{t}{\log \left(\frac{1}{t}\right)}\right)^{1 / 7} & \text { if } \frac{1}{k^{7 / 2} \log ^{5 / 2} k} \ll t \ll 1 .\end{cases}
$$


Note that the time windows in (7.2) and $(7.3)_{1}$ are not empty in view of the assumptions on $s_{0}$. Returning to the original variables and letting $M=1$ we obtain the early and moderate timescales in (2.1) and (2.2). Large timescales will be analyzed in the next section.

The rest of the section is devoted to showing that (5.9) and (5.10) imply (7.2) and (7.3). In Section 7.1 we show that, under (7.1), (5.9) and (5.10) are equivalent to

$$
\begin{gathered}
s^{\prime} \sim \frac{k}{s^{4}} \quad \text { if } s \ll \frac{1}{k} \quad \text { for } k \lesssim 1, \\
s^{\prime} \sim\left\{\begin{array}{ll}
\frac{k}{s^{4}} & \text { if } s^{2} \ll \frac{1}{k \log k}, \\
\frac{1}{s^{6} \log \left(\frac{1}{s}\right)} & \text { if } \frac{1}{k \log k} \ll s^{2} \ll 1,
\end{array} \text { for } k \gg 1 .\right.
\end{gathered}
$$

In Section 7.2 we easily infer (7.2) and (7.3) from (7.4) and (7.5).

7.1. The ODEs for $s$. Plugging (7.1) into (5.9), we obtain that if

$$
s \ll 1
$$

and

$$
s^{6} s^{\prime} \ll 1
$$

then

$$
\frac{1}{s^{6}} \sim \begin{cases}\left(\frac{s^{\prime}}{k}\right)^{3 / 2}+\frac{3}{2} s^{\prime} \log \left(\frac{s^{2} s^{\prime}}{k}\right) & \text { if } k \ll s^{2} s^{\prime} \text { and } k^{3} \ll s^{\prime}, \\ s^{\prime} \log \left(s^{3} s^{\prime}\right) & \text { if } 1 \ll s^{3} s^{\prime} \text { and } s^{\prime} \ll k^{3}\end{cases}
$$

The relation in (7.8) 1 may be split into two regimes:

$$
\frac{1}{s^{6}} \sim \begin{cases}\left(\frac{s^{\prime}}{k}\right)^{3 / 2} & \text { if }\left(\frac{s^{\prime}}{k^{3}}\right)^{1 / 2} \gg \log \left(\frac{s^{2} s^{\prime}}{k}\right), \\ s^{\prime} \log \left(\frac{s^{2} s^{\prime}}{k}\right) & \text { if }\left(\frac{s^{\prime}}{k^{3}}\right)^{1 / 2} \ll \log \left(\frac{s^{2} s^{\prime}}{k}\right) .\end{cases}
$$

Therefore (7.8) is equivalent to

$$
\frac{1}{s^{6}} \sim \begin{cases}\left(\frac{s^{\prime}}{k}\right)^{3 / 2} & \text { if } k \ll s^{2} s^{\prime} \text { and }\left(\frac{s^{\prime}}{k^{3}}\right)^{1 / 2} \gg \log \left(\frac{s^{2} s^{\prime}}{k}\right), \\ s^{\prime} \log \left(\frac{s^{2} s^{\prime}}{k}\right) & \text { if } k \ll s^{2} s^{\prime} \text { and } 1 \ll\left(\frac{s^{\prime}}{k^{3}}\right)^{1 / 2} \ll \log \left(\frac{s^{2} s^{\prime}}{k}\right), \\ s^{\prime} \log \left(s^{3} s^{\prime}\right) & \text { if } 1 \ll s^{3} s^{\prime} \text { and } s^{\prime} \ll k^{3}\end{cases}
$$

Plugging (7.1) into (5.10), we obtain

$$
\frac{1}{s^{6}} \sim\left(\frac{s^{\prime}}{k}\right)^{3 / 2} \quad \text { if } \alpha>0, s^{5} s^{\prime} \ll 1, \text { and } s \gg 1 .
$$

We now analyze each regime in (7.9) and (7.10).

- Within $(7.9)_{1}$, we have

$$
\frac{1}{s^{6}} \sim\left(\frac{s^{\prime}}{k}\right)^{3 / 2} \Longleftrightarrow s^{\prime} \sim \frac{k}{s^{4}} .
$$


Hence

$$
\begin{aligned}
k \ll s^{2} s^{\prime} & \stackrel{(7.11)}{\Longleftrightarrow} k \ll \frac{k}{s^{2}} \Longleftrightarrow(7.6), \\
\left(\frac{s^{\prime}}{k^{3}}\right)^{1 / 2} \gg \log \left(\frac{s^{2} s^{\prime}}{k}\right) & \stackrel{(7.11)}{\Longleftrightarrow} \frac{1}{k s^{2}} \gg \log \left(\frac{1}{s^{2}}\right) \\
& \Longleftrightarrow s^{2} \log \left(\frac{1}{s}\right) \ll \frac{1}{k},
\end{aligned}
$$

and (7.7) is absorbed by (7.6) and (7.12):

$$
s^{6} s^{\prime} \ll 1 \stackrel{(7.11)}{\Longleftrightarrow} s^{2} \ll \frac{1}{k} \stackrel{(7.6)}{\Longleftrightarrow}(7.12) .
$$

We now distinguish two cases. If $k \lesssim 1,(7.6)$ guarantees that (7.12) holds, and (7.4) follows for $s \ll 1$ (the window $1 \ll s \ll \frac{1}{k}$ in (7.4) will follow from (7.10)). If $k \gg 1$, we may rewrite the constraint in (7.12) as

$$
s^{2} \log \left(\frac{1}{s}\right) \ll \frac{1}{k} \Longleftrightarrow s^{2} \ll \frac{1}{k \log k} \ll 1 .
$$

Hence (7.13) enforces (7.6) and (7.5) ${ }_{1}$ follows.

- Within $(7.9)_{2}$ we have

$$
\frac{1}{s^{6}} \sim s^{\prime} \log \left(\frac{s^{2} s^{\prime}}{k}\right) \Longleftrightarrow \frac{1}{k s^{4}} \sim \frac{s^{2} s^{\prime}}{k} \log \left(\frac{s^{2} s^{\prime}}{k}\right)
$$

Then

$$
k s^{4} \ll 1 \Longleftrightarrow s^{2} s^{\prime} \gg k
$$

and in this case

$$
\begin{aligned}
\frac{1}{s^{6}} \sim s^{\prime} \log \left(\frac{s^{2} s^{\prime}}{k}\right) & \Longleftrightarrow \frac{s^{2} s^{\prime}}{k} \sim \frac{1}{k s^{4} \log \left(\frac{1}{k s^{4}}\right)} \\
& \Longleftrightarrow s^{\prime} \sim \frac{1}{s^{6} \log \left(\frac{1}{k s^{4}}\right)} .
\end{aligned}
$$

In particular,

$$
\begin{aligned}
\left(\frac{s^{\prime}}{k^{3}}\right)^{1 / 2} \ll \log \left(\frac{s^{2} s^{\prime}}{k}\right) & \stackrel{(7.15)}{\Longleftrightarrow} \frac{1}{k^{3} s^{6} \log \left(\frac{1}{k s^{4}}\right)} \ll \log ^{2}\left(\frac{1}{k s^{4} \log \left(\frac{1}{k s^{4}}\right)}\right) \\
& \stackrel{(7.14)}{\Longleftrightarrow} \frac{1}{k^{3} s^{6}} \ll \log ^{3}\left(\frac{1}{k s^{4}}\right) \\
& \stackrel{(7.14)}{\Longleftrightarrow} \frac{1}{k} \ll s^{2} \log \frac{1}{s^{2}} .
\end{aligned}
$$

Together with (7.6), (7.16) implies that $(7.9)_{2}$ is seen only if $1 \ll k$. In this case, the constraints in $(7.9)_{2}$ may be written as follows:

$$
1 \ll\left(\frac{s^{\prime}}{k^{3}}\right)^{1 / 2} \ll \log \left(\frac{s^{2} s^{\prime}}{k}\right) \stackrel{(7.16),(7.15)}{\Longleftrightarrow} \frac{1}{s^{2}} \log ^{1 / 3} \frac{1}{s} \ll \frac{1}{k s^{4}} \ll \frac{1}{s^{2}} \log \frac{1}{s}
$$




$$
\stackrel{(7.6)}{\Longleftrightarrow} \frac{1}{k^{3} \log ^{3} k} \ll s^{6} \ll \frac{1}{k^{3} \log k} .
$$

By (7.17) we deduce that $\log \left(\frac{1}{k s^{4}}\right) \sim \log \left(\frac{1}{s}\right)$. Therefore (7.15) reads as

$$
s^{\prime} \sim \frac{1}{s^{6} \log \left(\frac{1}{s}\right)},
$$

and holds provided (7.6), (7.7), (7.14), and (7.18) are satisfied. Noting that (7.6) is implied by (7.18) (since $k \gg 1$ ) and that

$$
\begin{aligned}
(7.7) & \Longleftrightarrow s^{6} s^{\prime} \ll 1 \stackrel{(7.19)}{\Longleftrightarrow}(7.6), \\
(7.14) & \Longleftrightarrow k s^{4} \ll 1 \Longleftrightarrow k^{3 / 2} s^{6} \ll 1 \Longleftarrow(7.18),
\end{aligned}
$$

we conclude that

$$
s^{\prime} \sim \frac{1}{s^{6} \log \left(\frac{1}{s}\right)} \quad \text { if } \frac{1}{k^{3} \log ^{3} k} \ll s^{6} \ll \frac{1}{k^{3} \log k} \quad \text { and } k \gg 1 .
$$

- For $(7.9)_{3}$ we argue exactly as in Section 6; we obtain that (7.19) holds provided $s^{\prime} \ll k^{3}$ and (7.6) are satisfied. Now

$$
s^{\prime} \ll k^{3} \Longleftrightarrow \frac{1}{k^{3}} \ll s^{6} \log \left(\frac{1}{s}\right) .
$$

Because of (7.6), also $(7.9)_{3}$ is seen only if $1 \ll k$, and in this case

$$
s^{\prime} \ll k^{3} \Longleftrightarrow s^{6} \gg \frac{1}{k^{3} \log k} .
$$

Combining (7.6), (7.20), and (7.21) we obtain $(7.5)_{2}$.

- Within (7.10), we have

$$
s^{\prime} \sim \frac{k}{s^{4}} \quad \text { if } \quad \alpha>0, s^{5} s^{\prime} \ll 1 \text { and } s \gg 1
$$

Since

$$
s^{5} s^{\prime} \sim k s \ll 1 \Longleftrightarrow s \ll \frac{1}{k},
$$

the regime in (7.10) is not empty only if $k \ll 1$, and (7.4) follows for $1 \ll s \ll \frac{1}{k}$.

7.2. The timescales. We now infer from (7.4) and (7.5) the scaling laws for $s$ given by $(7.2)$ and $(7.3)$.

(I) If $k \lesssim 1$ and $s_{0} \ll 1 / k$, it follows from (7.4) that

$$
s^{5} \sim s_{0}^{5}+5 k t \sim k t \quad \text { provided } t \gg \frac{s_{0}^{5}}{k},
$$

and

$$
s \ll \frac{1}{k} \Longleftrightarrow t \ll \frac{1}{k^{6}},
$$

whence (7.2). 
(II) If $k \gg 1$, we assume that $s_{0}^{2} \ll \frac{1}{k \log k}$, so that both regimes in (7.5) are seen. According to $(7.5)_{1}$, we have

$$
s^{5} \sim s_{0}^{5}+5 k t \sim k t \quad \text { provided } t \gg \frac{s_{0}^{5}}{k},
$$

which holds as long as

$$
s^{2} \ll \frac{1}{k \log k} \stackrel{(7.22)}{\Longleftrightarrow} t \ll\left(\frac{1}{k^{7} \log ^{5} k}\right)^{1 / 2}=: t_{1} .
$$

As $t \sim t_{1}$, the free boundary enters the second regime in (7.5), which has already been analyzed in Section 6; it follows from (6.6) that

$$
s(t) \sim\left(\frac{t}{\log \left(\frac{1}{t}\right)}\right)^{1 / 7} \quad \text { if } \max \left\{t_{1}, s_{1}^{7} \log \left(\frac{1}{s_{1}}\right)\right\} \ll t \ll 1 \quad \text { and } \quad s_{1} \ll 1,
$$

with initial condition $s_{1}:=s\left(t_{1}\right)=\left(k t_{1}\right)^{1 / 5}$. Note that $s_{1} \ll 1$ since $k \gg 1$ and $t_{1}$ is given by (7.23). Since

$$
s_{1}^{7} \log \left(\frac{1}{s_{1}}\right) \stackrel{(7.5)}{\sim} \frac{s_{1}^{5}}{k} \stackrel{(7.22)}{\sim} t_{1}
$$

the lower bounds on $t$ in (7.24) coincide. Therefore we conclude that

$$
s(t) \sim\left(\frac{t}{\log \left(\frac{1}{t}\right)}\right)^{1 / 7} \quad \text { if } t_{1} \ll t \ll 1 .
$$

Gathering (7.22), (7.23), and (7.25) we obtain (7.3).

\section{Long time scaling laws in complete wetting}

The asymptotic of this section is based on two main assumptions:

(I) the timescale is "large";

(II) the evolution is "quasi-selfsimilar".

We will argue that

$$
s(t) \sim t^{1 / 6} \quad \text { if } t \gg \max \left\{1, \frac{1}{k^{6}}\right\} .
$$

Comparing (8.1) with (7.2) and (7.3), we see that the whole remaining range of timescales is covered by (8.1). In terms of the original variables, we obtain the final timescale in (2.1) and (2.2).

We now motivate (8.1). In complete wetting, $h \rightarrow 0$ as $t \rightarrow+\infty$; hence $h \ll 1$ everywhere for sufficiently large times, and conservation of mass implies that

$$
s \gg 1,
$$

which partially encodes (I). Since $h^{3}+h^{2} \sim h^{2}$ everywhere, we may replace the equation in (3.1) with

$$
h_{t}+\left(h^{2} h_{x x x}\right)_{x}=0
$$


Following (I), we introduce the selfsimilar variable transformation of (8.3) which preserves mass:

$$
h=t^{-1 / 6} f(t, y), \quad y=x t^{-1 / 6} \in(0, a(t)), \quad \text { where } a(t)=t^{-1 / 6} s(t) .
$$

Then

$$
\left\{\begin{array}{l}
\frac{1}{6}(y f)_{y}-t f_{t}=\left(f^{2} f_{y y y}\right)_{y}, \quad f>0 \quad \text { in }(0, a), \\
\left.f_{y}\right|_{y=0}=\left.f_{y y y}\right|_{y=0}=0,\left.f\right|_{y=a}=0, \int_{0}^{a} f \mathrm{~d} y=1 / 2
\end{array}\right.
$$

while the boundary condition reads as

$$
\left.f_{y}^{2}\right|_{y=a}=\frac{1}{k t^{1 / 6}}\left(\lim _{y \rightarrow a(t)} f f_{y y y}\right) .
$$

Since (8.5) is not time independent, an exact selfsimilar profile does not exist. However, if

$$
k t^{1 / 6} \gg 1
$$

(which completes (I)), the contact-angle condition is only a perturbation of $\left.f_{y}\right|_{y=a}=0$. Hence we assume that $f$ is quasi-selfsimilar in the sense that it has an expansion of the form

$$
f(t, y)=f_{0}(y)+\left(k^{6} t\right)^{-1} f_{1}(y)+\ldots,
$$

which encodes (II). Then, at leading order, (8.4) reads as

$$
\left\{\begin{array}{l}
\frac{1}{6} y f_{0}=f_{0}^{2} f_{0 y y y}, \quad f>0 \quad \text { in }(0, a), \\
\left.f_{0 y}\right|_{y=0}=\left.f_{0 y y y}\right|_{y=0}=0,\left.f_{0}\right|_{y=a}=0,\left.f_{0 y}\right|_{y=a}=0, \int_{0}^{a} f_{0}(y) \mathrm{d} y=1 / 2 .
\end{array}\right.
$$

As is well-known [1], (8.7) has a unique solution $\left(f_{0}, a\right)$. Therefore, recalling $(8.2)$ and (8.6), we obtain (8.1).

\section{Partial wetting with contact line friction}

In the case of partial wetting, $\alpha_{S}>0$, the profile of a spreading droplet converges to the unique steady state with mass 1 and contact angles $\alpha_{S}$ as $t \rightarrow+\infty$ :

$$
h \rightarrow \frac{3}{4 s_{\infty}^{3}}\left(s_{\infty}^{2}-x^{2}\right)_{+}, s \uparrow s_{\infty}=\sqrt{\frac{3}{2 \alpha_{S}}}, s^{\prime} \rightarrow 0 \quad \text { as } t \rightarrow+\infty .
$$

We focus on the most interesting case of

$$
\alpha_{S} \gg 1 \stackrel{(9.1)}{\Longleftrightarrow} s \leq s_{\infty} \ll 1,
$$

which guarantees the persistence for all times of a macroscopic profile. Because of $s \ll 1$, (5.10) may be ignored and we only have to look at (5.9), which we rewrite for the reader's convenience:

$$
\left(\frac{3}{2 s^{2}}\right)^{3} \sim \begin{cases}\alpha^{3}+3 s^{\prime} \log (s \alpha) & \text { if } 1 \ll s \alpha \text { and } s^{\prime} \ll \alpha^{3} \\ 3 s^{\prime} \log \left(s\left(s^{\prime}\right)^{1 / 3}\right) & \text { if } 1 \ll s^{3} s^{\prime} \text { and } s^{\prime} \gg \alpha^{3} .\end{cases}
$$


In view of (9.1), for sufficiently large times (9.3) reduces to

$$
\left(\frac{3}{2 s^{2}}\right)^{3} \sim \alpha_{S}^{3}+3 s^{\prime} \log \left(s \alpha_{S}\right)
$$

which is equivalent to the well-known Cox-Hocking relation between the effective and the microscopic contact angle. In terms of the original variables, it coincides with (1.10).

The relation (9.4) yields an exponential convergence of $s$ to $s_{\infty}$. Indeed, let

$$
s=\sqrt{\frac{3}{2 \alpha_{S}}} \hat{s}, \quad t=\sqrt{\frac{27}{2 \alpha_{S}^{7}}} \log \alpha_{S} \hat{t} .
$$

In view of $(9.1)$ and $(9.2), \log \left(\alpha_{S} s\right) \sim \log \left(\sqrt{\alpha_{S}}\right)$ as $t \rightarrow+\infty$. Hence (9.4) reads as

$$
\frac{\mathrm{d} \hat{s}}{\mathrm{~d} \hat{t}} \sim \frac{1-\hat{s}^{6}}{\hat{s}^{6}} .
$$

An integration shows that $1-\hat{s}(\hat{t}) \sim e^{-6 \hat{t}}$ as $\hat{t} \rightarrow+\infty$, i.e.

$$
\sqrt{\frac{3}{2 \alpha_{S}}}-s(t) \sim e^{-D t} \quad \text { as } t \rightarrow+\infty, \quad D:=\sqrt{\frac{8 \alpha_{S}^{7}}{3 \log ^{2} \alpha_{S}}} .
$$

In order to infer the timescale of validity of (9.4), we have to give a closer look to (9.3) in order to identify the intermediate scaling laws which precede (9.4). We will argue that

(i) if $|k \log k| \lesssim \alpha_{S}$, then

$$
s(t) \sim(k t)^{1 / 5} \quad \text { for } \quad \frac{s_{0}^{5}}{k} \ll t \ll \frac{1}{k \alpha_{S}^{5 / 2}} ;
$$

(ii) if $k \lesssim \alpha_{S} \ll k \log k$, then

$$
s(t) \sim \begin{cases}(k t)^{1 / 5} & \text { if } \frac{s_{0}^{5}}{k} \ll t \ll \frac{1}{k^{7 / 2} \log ^{5 / 2} k}, \\ \left(\frac{t}{\log \left(\frac{1}{t}\right)}\right)^{1 / 7} & \text { if } \frac{1}{k^{7 / 2} \log ^{5 / 2} k} \ll t \ll \frac{1}{k^{7 / 6} \alpha_{S}^{7 / 3} \log ^{1 / 6}\left(\alpha_{S}^{2} k\right)}=: t_{2} .\end{cases}
$$

(iii) if $\alpha_{S} \ll k$, then

$$
s(t) \sim \begin{cases}(k t)^{1 / 5} & \text { if } \frac{s_{0}^{5}}{k} \ll t \ll \frac{1}{k^{7 / 2} \log ^{5 / 2} k}, \\ \left(\frac{t}{\log \left(\frac{1}{t}\right)}\right)^{1 / 7} & \text { if } \frac{1}{k^{7 / 2} \log ^{5 / 2} k} \ll t \ll \frac{1}{\alpha_{S}^{7 / 2} \log ^{1 / 6} \alpha_{S}} .\end{cases}
$$

Preliminarily we observe that

$$
\alpha=\sqrt{\frac{s^{\prime}}{k}+\alpha_{S}^{2}} \sim \begin{cases}\left(\frac{s^{\prime}}{k}\right)^{1 / 2} & \text { if } s^{\prime} \gg k \alpha_{S}^{2}, \\ \alpha_{S} & \text { if } s^{\prime} \ll k \alpha_{S}^{2} .\end{cases}
$$


Because of (9.5), (9.3) coincides with the case of complete wetting as long as $s^{\prime} \gg$ $k \alpha_{S}^{2}$. Therefore (7.2) and (7.3) hold under the additional constraints that $s \ll 1$ and $s^{\prime} \gg k \alpha_{S}^{2}$; imposing them, a few simple computations yield (i), (ii), and (iii) up to $t=t_{2}$. When $s^{\prime} \ll k \alpha_{S}^{2}$, then $\alpha \sim \alpha_{S}$ and (9.3) $)_{1}$ coincides with (9.4). Instead, (9.3) yields

$$
s(t) \sim\left(\frac{t}{\log \left(\frac{1}{t}\right)}\right)^{1 / 7} \quad \text { if } \frac{1}{k^{7 / 2} \log ^{5 / 2} k} \ll t \ll 1,
$$

with the additional constraints that $\alpha_{S}^{3} \ll s^{\prime} \ll k \alpha_{S}^{2}$ and that $s^{3} s^{\prime} \gg 1$. Hence this regime is seen only if $\alpha_{S} \ll k$; in this case, a few more computations imposing the bounds on the speed yield (iii).

\section{REFERENCES}

[1] F. Bernis, L.A. Peletier, and S.M. Williams, Source type solutions of a fourth order nonlinear degenerate parabolic equation, Nonlin. Anal., 18(3), 217-234, 1992.

[2] M. Bertsch, R. Dal Passo, S.H. Davis, and L. Giacomelli, Effective and microscopic contact angles in thin film dynamics, Euro. J. Appl. Math., 11(2), 181-201, 2000.

[3] M. Bertsch, L. Giacomelli, and G. Karali, Thin-film equations with "partial wetting" energy: Existence of weak solutions, Phys. D, 209(1-4), 17-27, 2005.

[4] S. Boatto, L.P. Kadanoff, and P. Olla, Traveling-wave solutions to thin-film equations, Phys. Rev. E (3), 48(6), 4423-4431, 1993.

[5] D. Bonn, J. Eggers, J. Indekeu, J. Meunier, and E. Rolley, Wetting and spreading, Rev. Mod. Phys., 81(2), 739-805, 2009.

[6] R. Buckingham, M. Shearer, and A. Bertozzi, Thin film traveling waves and the Navier slip condition, SIAM J. Appl. Math., 63(2), 722-744, 2002.

[7] M. Chiricotto and L. Giacomelli, Droplets spreading with contact-line friction: Lubrication approximation and traveling wave solutions, Commun. Appl. Ind. Math., 2(2), 1-16, 2011.

[8] R.G. Cox, The dynamics of the spreading of liquids on a solid surface. 1. Viscous-flow, J. Fluid Mech., 168, 169-194, 1986.

[9] P.G. de Gennes, Wetting - statics and dynamics, Rev. Mod. Phys., 57(3), 827-863, 1985.

[10] E.B. Dussan V. and S.H. Davis, On the motion of fluid-fluid interface along a solid surface, J. Fluid Mech., 65, 71-95, 1974.

[11] P. Ehrhard and S.H. Davis, Nonisothermal spreading of liquid drops on horizontal plates, J. Fluid Mech., 229, 365-388, 1991.

[12] L. Giacomelli and F. Otto, Droplet spreading: Intermediate scaling law by PDE methods, Comm. Pure Appl. Math., 55(2), 217-254, 2002.

[13] L. Giacomelli and F. Otto, Rigorous lubrication approximation, Interfaces Free Bound., 5(4), 483-529, 2003.

[14] K.B. Glasner, Variational models for moving contact lines and the quasi-static approximation, Euro. J. Appl. Math., 16(6), 713-740, 2005.

[15] H.P. Greenspan, On the motion of a small viscous droplet that wets a surface, J. Fluid Mech., 84, 125-143, 1978.

[16] P.J. Haley and M.J. Miksis, The effect of the contact line on droplet spreading, J. Fluid Mech., $223,57-81,1991$.

[17] L.M. Hocking, The spreading of a thin drop by gravity and capillarity, Q.J. Mech. Appl. Math., 36, 55-69, 1983.

[18] L.M. Hocking, Rival contact-angle models and the spreading of drops, J. Fluid Mech., 239, 671-681, 1992.

[19] C. Huh and L.E. Scriven, Hydrodynamic model of steady movement of a solid/liquid/fluid contact line, J. Colloid Interface Sci., 35, 85-101, 1971.

[20] H. Knüpfer and N. Masmoudi, Darcy flow on a plate with prescribed contact angle - wellposedness and lubrication approximation, preprint, 2010.

[21] A. Oron, S.H. Davis, and S.G. Bankoff, Long-scale evolution of thin liquid films, Rev. Mod. Phys., 69, 931-980, 1997.

[22] T.Z. Qian, X.P. Wang, and P. Sheng, Molecular scale contact line hydrodynamics of immiscible flows, Phys. Rev. E, 68(1), Part 2, 016306, 2003. 
[23] W. Ren and W. E, Boundary conditions for the moving contact line problem, Phys. Fluids, 19, 022101, 2007.

[24] W. Ren and W. E, Derivation of continuum models for the moving contact line problem based on thermodynamic principles, Comm. Math. Sci., 9(2), 597-606, 2011.

[25] W. Ren, D. Hu, and W. E, Continuum models for the contact line problem, Phys. Fluids, 22, 102103, 2010.

[26] L.H. Tanner, The spreading of silicone oil drops on horizontal surfaces, J. Phys. D: Appl. Phys., 12(9), 1473, 1979.

[27] P.A. Thompson and S.M. Troian, A general boundary condition for liquid flow at solid surfaces, Nature, 389(6649), 360-362, 1997. 